\title{
Prognostic Model for Survival and Recurrence in Patients with Early-Stage Cervical Cancer: A Korean Gynecologic Oncology Group Study (KGOG 1028)
}

\author{
E Sun Paik, MD, $\mathrm{PhD}$ \\ Myong Cheol Lim, MD, PhD² \\ Moon-Hong Kim, MD, PhD \\ Yun Hwan Kim, MD, PhD ${ }^{4}$ \\ Eun Seop Song, MD, PhD 5 \\ Seok Ju Seong, MD, PhD \\ Dong Hoon Suh, MD, PhD \\ Jong-Min Lee, MD, PhD ${ }^{8}$ \\ Chulmin Lee, MD, $\mathrm{PhD}^{9}$ \\ Chel Hun Choi, MD, PhD
}

*A list of author's affiliations appears at the end of the paper.

\section{Correspondence: Chel Hun Choi, MD, PhD Department of Obstetrics and Gynecology, Samsung Medical Center, Sungkyunkwan University School of Medicine, 81 Irwon-ro, Gangnam-gu, Seoul 06351, Korea \\ Tel: 82-2-3410-3545 \\ Fax: 82-2-3410-0630 \\ E-mail: chelhun.choi@samsung.com}

Received March 5, 2019

Accepted August 3, 2019

Published Online August 5, 2019

${ }^{*}$ E Sun Paik and Myong Cheol Lim contributed equally to this work.

*Presented April 27, 2018, in the plenary session of the 33rd Annual Meeting of the Korean Society of Gynecologic Oncology, Jeju, Korea.

\begin{abstract}
Purpose
We aimed to develop and validate individual prognostic models in a large cohort of cervical
\end{abstract} cancer patients that were primarily treated with radical hysterectomy.

\section{Materials and Methods}

We analyzed 1,441 patients with early-stage cervical cancer treated between 2000 and 2008 from the Korean Gynecologic Oncology Group multi-institutional cohort: a train cohort $(n=788)$ and a test cohort $(n=653)$. Models predicting the risk for overall survival (OS), disease-free survival (DFS), lymphatic recurrence and hematogenous recurrence were developed using Cox analysis and stepwise backward selection and best-model options. The prognostic performance of each model was assessed in an independent patient cohort. Model-classified risk groups were compared to groups based on traditional risk factors.

\section{Results}

Independent risk factors for OS, DFS, lymphatic recurrence, and hematogenous recurrence were identified for prediction model development. Different combinations of risk factors were shown for each outcome with best predictive value. In train cohort, area under the curve (AUC) at 2 and 5 years were $0.842 / 0.836$ for recurrence, and 0.939/0.882 for OS. When applied to a test cohort, the model also showed accurate prediction result (AUC at 2 and 5 years were $0.799 / 0.723$ for recurrence, and $0.844 / 0.806$ for OS, respectively). The Kaplan-Meier plot by proposed model-classified risk groups showed more distinctive survival differences between each risk group.

\section{Conclusion}

We developed prognostic models for OS, DFS, lymphatic and hematogenous recurrence in patients with early-stage cervical cancer. Combining weighted clinicopathologic factors, the proposed model can give more individualized predictions in clinical practice.

\section{Introduction}

Cervical cancer is the fourth most frequent malignancy in women and the seventh overall worldwide [1]. In Korea, it is one of the common gynecologic cancers, representing $9.8 \%$ of newly diagnosed malignancies in women [2]. Although radical hysterectomy has been demonstrated as a standard treatment for International Federation of Gynecology and Obstetrics (FIGO) stage IA-IIA uterine cervix cancer patients,

\section{Key words}

Disease-free survival, Prognostic factor, Prognoses,

Survival analyses, Uterine cervical neoplasms 


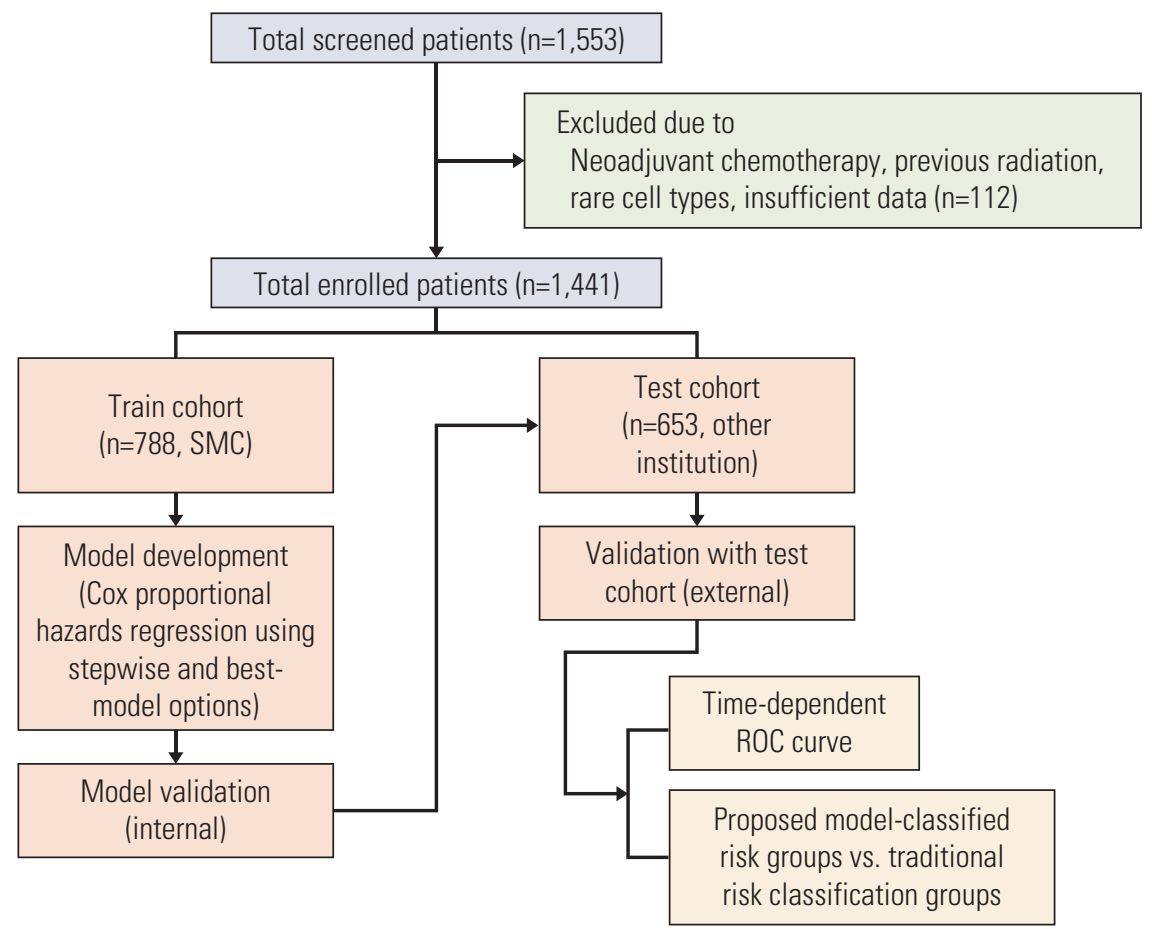

Fig. 1. Flowchart of included patients. SMC, Samsung Medical Center; ROC, receiver operating characteristic.

powerful prognostic parameter, other clinicopathological parameters have been shown to have additional significance in predicting patient prognosis [8]. Certain clinical variables including pelvic lymph node metastases, parametrial involvement, positive surgical margins, tumor size, deep stromal invasion, and lymphovascular space invasion (LVSI) are used for prediction of recurrence or survival. Information about these variables aids in determining the appropriate recommendation for adjuvant therapy $[9,10]$. While some of the factors are considered to be somewhat controversial, others are widely accepted prognostic factors.

Traditionally, adjuvant therapy was given on the basis of risk factors. Combinations of factors have been shown to be more reliable than single factors [11-14]. However, there has been no prior consensus about the relative importance of different prognostic markers for outcomes including overall survival (OS) and disease-free survival (DFS) for lymphatic and hematogenous recurrences. Models developed for the prediction of recurrence or survival have performed well in the populations in which they were designed, but few models have been externally validated [15-18]. In addition, there are no published independent models separately predicting OS and DFS for lymphatic and hematogenous recurrences.

The current study aims to develop and validate a prognostic model of early-stage cervical cancer patients using a large study cohort to provide more individualized predictions in clinical practice.

\section{Materials and Methods}

\section{Patients}

After obtaining approval from the local institutional review board, the medical records of the patients with earlystage cervical cancer from January 2000 to December 2008 were retrospectively reviewed from nine Korean Gynecologic Oncology Group (KGOG)-affiliated institutions. Patients were included if they had pathologically confirmed cervical cancer, a clinical diagnosis of FIGO stage IB-IIA disease, and who had undergone radical hysterectomy (type II or III) with pelvic and/or para-aortic lymphadenectomy. Patients treated with neoadjuvant chemotherapy before surgery, those with previous radiation therapy, those with incidentally found cervical cancer after simple hysterectomy, and those with rare cell types were excluded. Patients with insufficient data were also excluded from analysis. From a database of 1,553 patients, 1,441 satisfied the eligibility criteria. Before analysis, patients were allocated based on treatment institution to one of two groups: a train cohort $(\mathrm{n}=788$ 
Table 1. Demographic and pathologic data of the train and test cohorts

\begin{tabular}{|c|c|c|c|c|}
\hline Characteristic & $\begin{array}{l}\text { All patients } \\
(\mathrm{n}=1,441)\end{array}$ & $\begin{array}{l}\text { Train cohort } \\
\quad(\mathbf{n}=788)\end{array}$ & $\begin{array}{c}\text { Test cohort } \\
(n=653)\end{array}$ & p-value \\
\hline Age (yr) & $48(40-58)$ & $48(40-57)$ & $48(41-58)$ & 0.514 \\
\hline BMI & $23.4(21.4-25.8)$ & $23.4(21.4-25.5)$ & $23.5(21.4-25.8)$ & 0.269 \\
\hline \multicolumn{5}{|l|}{ FIGO stage } \\
\hline IB1 & $1,085(75.3)$ & $628(79.7)$ & $457(70.0)$ & $<0.001$ \\
\hline IB2 & 167 (11.6) & $63(8.0)$ & $104(15.9)$ & \\
\hline IIA & $189(13.1)$ & $97(12.3)$ & $92(14.1)$ & \\
\hline \multicolumn{5}{|l|}{ Histology } \\
\hline SCC & $1,089(75.6)$ & $587(74.5)$ & $502(76.9)$ & 0.324 \\
\hline Adenocarcinoma & $352(24.4)$ & $201(25.5)$ & $151(23.1)$ & \\
\hline Tumor size (cm) & $2.7(1.5-4.0)$ & $2.5(1.5-4.0)$ & $2.8(1.9-3.8)$ & 0.028 \\
\hline \multicolumn{5}{|l|}{ Lymphovascular space invasion } \\
\hline Negative & $791(54.9)$ & $453(61.7)$ & $338(54.6)$ & 0.010 \\
\hline Positive & $562(39.1)$ & $281(38.3)$ & $281(45.4)$ & \\
\hline \multicolumn{5}{|l|}{ Invasion depth } \\
\hline Inner $1 / 3$ & $445(30.8)$ & $266(33.8)$ & $179(29.2)$ & 0.030 \\
\hline Middle $1 / 3$ & $380(26.4)$ & $193(24.5)$ & $187(30.5)$ & \\
\hline Deep $1 / 3$ & $577(40.0)$ & $329(41.8)$ & $248(40.4)$ & \\
\hline \multicolumn{5}{|l|}{ Lymph node metastasis } \\
\hline Negative & $1,177(81.7)$ & $637(80.8)$ & $540(83.1)$ & 0.544 \\
\hline Pelvic LN positive & $239(16.6)$ & $138(17.5)$ & $101(15.5)$ & \\
\hline Para-aortic LN positive & $21(1.5)$ & $12(1.6)$ & $9(1.4)$ & \\
\hline \multicolumn{5}{|l|}{ Parametrial involvement } \\
\hline Negative & $1,323(91.8)$ & $716(90.9)$ & $607(93.2)$ & 0.099 \\
\hline Positive & $116(8.2)$ & $72(9.1)$ & $44(6.8)$ & \\
\hline \multicolumn{5}{|l|}{ Resection margin } \\
\hline Negative & $1,381(95.8)$ & $770(97.7)$ & $611(93.6)$ & $<0.001$ \\
\hline CIN positive & $16(1.1)$ & 0 & $16(2.5)$ & \\
\hline Cancer positive & $44(3.1)$ & $18(2.3)$ & $26(4.0)$ & \\
\hline \multicolumn{5}{|l|}{ Surgical method } \\
\hline Laparotomy & $1,267(87.9)$ & $710(90.1)$ & $557(85.4)$ & 0.008 \\
\hline Laparoscopy & $173(12.1)$ & $78(9.9)$ & $95(14.6)$ & \\
\hline Serum SCC level (ng/mL) & $1.2(0.7-2.5)$ & $1.2(0.7-2.4)$ & $1.2(0.7-2.5)$ & 0.693 \\
\hline Pretreatment serum hemoglobin level $(\mathrm{g} / \mathrm{dL})$ & $12.7(11.8-13.5)$ & $12.7(11.8-13.4)$ & $12.8(11.9-13.5)$ & 0.067 \\
\hline Pretreatment serum platelet level $\left(\times 10^{3} / \mu \mathrm{L}\right)$ & $252(211.5-299.0)$ & $250.0(211.5-296.5)$ & $255.5(218.0-299.0)$ & 0.140 \\
\hline
\end{tabular}

Values are presented as median (range) or number (\%). BMI, body mass index; FIGO, International Federation of Gynecoloy and Obstetrics; LN, lymph node; CIN, cervical intraepithelial neoplasia; SCC, squamous cell carcinoma.

from Samsung Medical Center) and a test cohort ( $\mathrm{n}=653$ from other institutions) (Fig. 1).

\section{Selection of prognostic variables for survival and recur- rence}

To identify variables predicting survival, the following factors were tested: age, body mass index (BMI), histology, FIGO stage, tumor size, parametrial invasion (PMI), LVSI, depth of invasion (DI), lymph node metastasis (LNM, pelvic and/or para-aortic), positive resection margin (RM), pretreatment serum squamous cell carcinoma (SCC) antigen $(\mathrm{Ag})$, hemoglobin $(\mathrm{Hb})$, platelet level, and surgical method (laparotomy vs. laparoscopy). Age, BMI, tumor size, serum $\mathrm{SCC} \mathrm{Ag,} \mathrm{Hb}$, and platelet level were considered continuous variables. FIGO stage, histology, PMI, LVSI, DI, LMN status (pelvic and para-aortic), positive RM invasion, and surgical method (laparotomy vs. laparoscopy) were considered categorical variables. The associations between clinicopathologic parameters were examined using the chi-square test, Fisher 
exact test, and the Pearson correlation test, as appropriate. DFS and OS were estimated using the Kaplan-Meier method and log-rank test. DFS was defined as the time interval from surgery to the first evidence of any recurrence or last followup. DFS to lymphatic recurrence and/or hematogenous recurrence was defined as the time interval from surgery to the first evidence of lymphatic and / or hematogenous recurrence, respectively. OS was described as the time interval from diagnosis to date of death or last follow-up.

\section{Model development}

A Cox proportional hazards regression analysis was performed on each of the individual factors for DFS, OS, hematogenous recurrence, and lymphatic recurrence to estimate individual risk ratios. Cox proportional hazards regression using stepwise and best-model options (Akaike's information criterion) identified the best combinations as predictors and obtained adjusted risk ratios. Variables with a p-value $<0.05$ in the univariate analysis were entered as candidate variables into a stepwise regression model (conditional backward selection was used to avoid sparse-data biases) [19]. The selected model was applied to original data, and the final model was selected with exclusion of factors with p-value of $>0.05$. We then calculated a risk score (RS) for each patient, which is the sum of the estimated coefficients multiplied by the value of the factors on the training set. We used this formula to compute RS on the test set.

\section{Model validation}

For validation of the survival prediction model, we plotted the negative $\log \mathrm{p}$-values of the log-rank test using all possible cutoffs of RS from each model in both train and test cohort. In addition, we also constructed time-dependent receiver operating characteristic (ROC) curves using the Nearest Neighbor Estimation method and area under the curve (AUC) in both cohorts [20]. Last, we compared the traditional risk classification (e.g., low risk, intermediate risk, and high risk) to our risk model. Traditional risk was as follows: any one of LNM, PMI, positive RM is considered high risk, two or more of the following risk as intermediate risk; LVSI positive, more than DI $1 / 2$, and tumor size of $4 \mathrm{~cm}$, and the others were classified low-risk group. For the comparison, we also categorized the patients into three groups according to RSs of proposed model. The cutoffs between three groups were arbitrarily chosen by the distribution of the score. Kaplan-Meier survival curve was plotted according to each group.

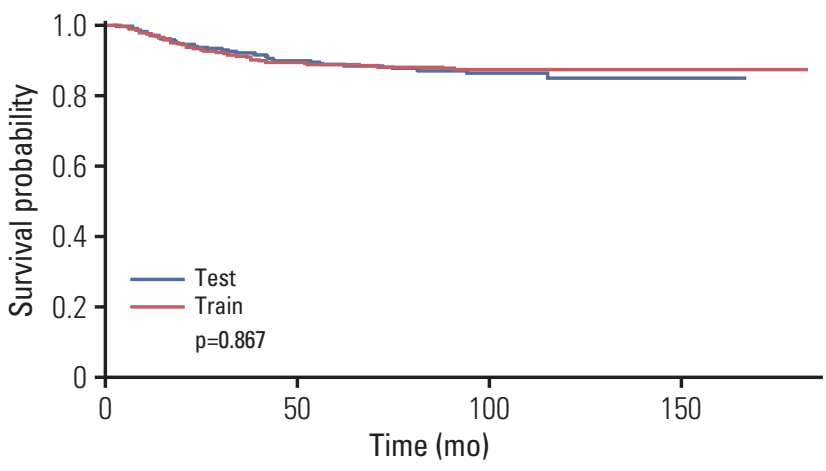

Test $653567511462407336259196138 \quad 97 \quad 5523 \quad 13 \quad 5 \quad 0$ Train $78872965059754343432326420215610778 \quad 4931 \quad 17 \quad 5$

Fig. 2. Survival comparison of train and test cohort.

\section{Statistical analysis}

Statistical analysis was performed using SAS ver. 9.4 (SAS Institute, Cary, NC) and R 3.3.2 (Vienna, Austria; http:// www.R-project.org/). A p-value of $<0.05$ was considered significant.

\section{Ethical statement}

All medical records were collected according to the protocol and case report forms approved by each institutional review board, which waived the requirement for informed consent.

\section{Results}

Patient characteristics are listed in Table 1. Total of 1,441 patients were included, and there were significant differences in the stage, LVSI, resection margin, surgical method, and tumor size between train cohort $(\mathrm{n}=788)$ and test cohort $(\mathrm{n}=653)$. After a median follow-up period of 63.7 months (range, 3.0 to 183.3 months), 134 patients experienced recurrences, and there were 72 deaths. The 5 -year recurrence rates were $91.8 \%$ and $92.1 \%$ in the train and test cohorts, respectively ( $\mathrm{p}=0.867)$ (Fig. 2).

\section{Model development for prediction of survival and recur- rence}

Cox proportional hazards regression using stepwise and best-model options yielded statistically significant predictors 


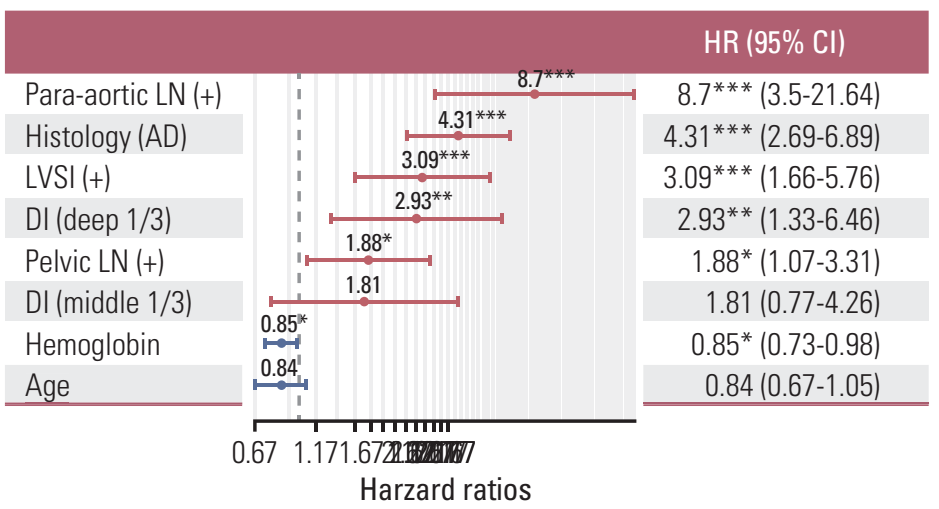

B

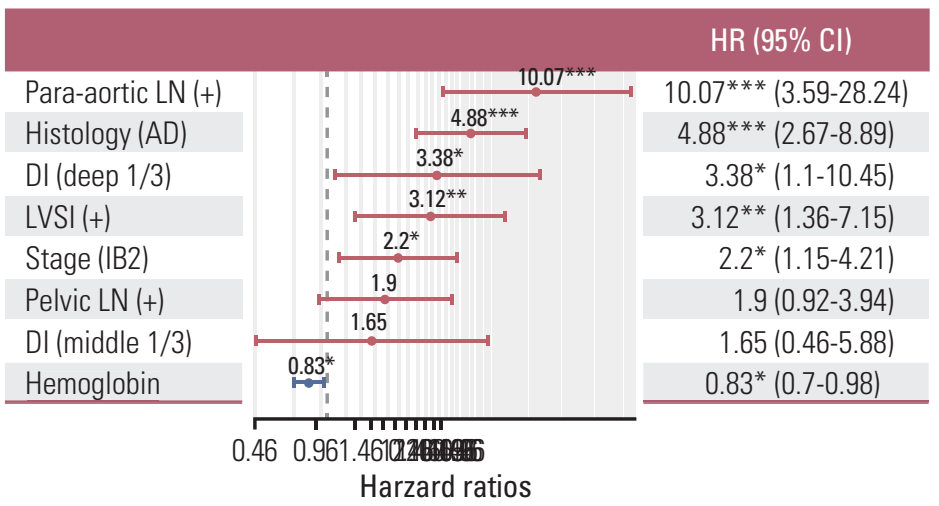

C

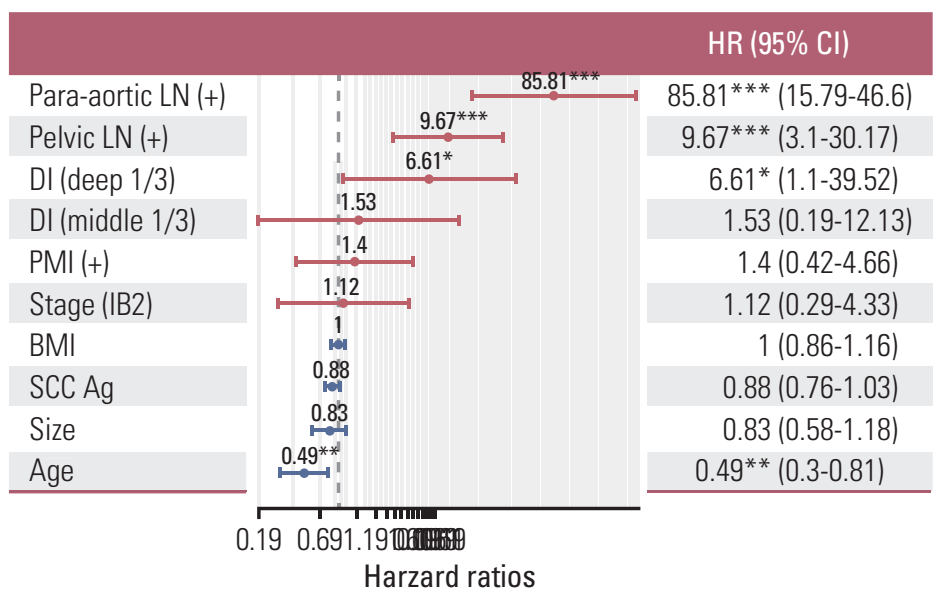

Fig. 3. Stepwise Cox proportional hazards regression analysis for disease-free survival (A), overall survival (B), lymphatic recurrence (C), and hematogenous recurrence (D) for prognostic model. HR, hazard ratio; CI, confidence interval; LN, lymph node; AD, adenocarcinoma; LVSI, lymphovascular space invasion; DI, depth of invasion; PMI, parametrial invasion; BMI, body mass index; SCC Ag, SCC, squamous cell carcinoma antigen. ${ }^{*} \mathrm{p}<0.05,{ }^{* *} \mathrm{p}<0.01$, ${ }^{* * *} \mathrm{p}<0.001$. (Continued to the next page) 


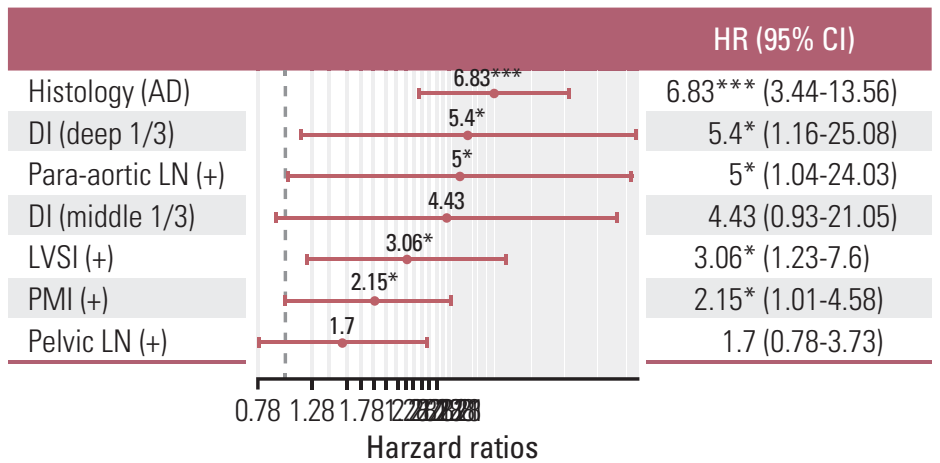

Fig. 3. (Continued from the previous page)

A

Color key

and histogram
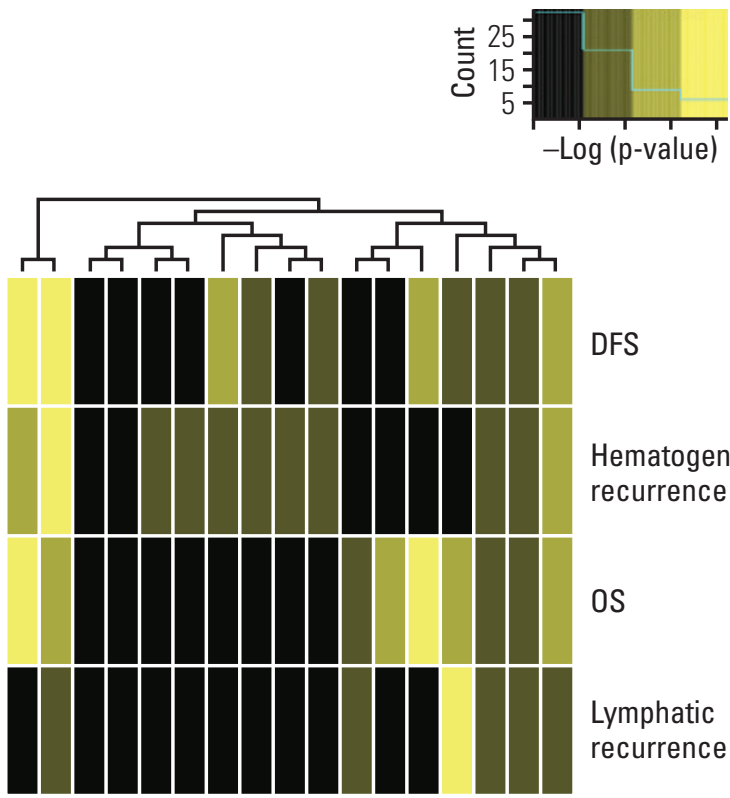

DFS

Hematogenous

recurrence

OS

Lymphatic recurrence

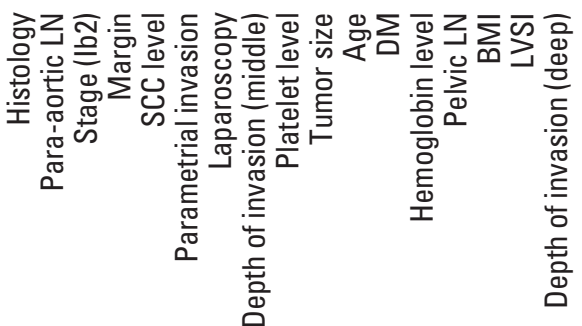

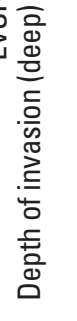

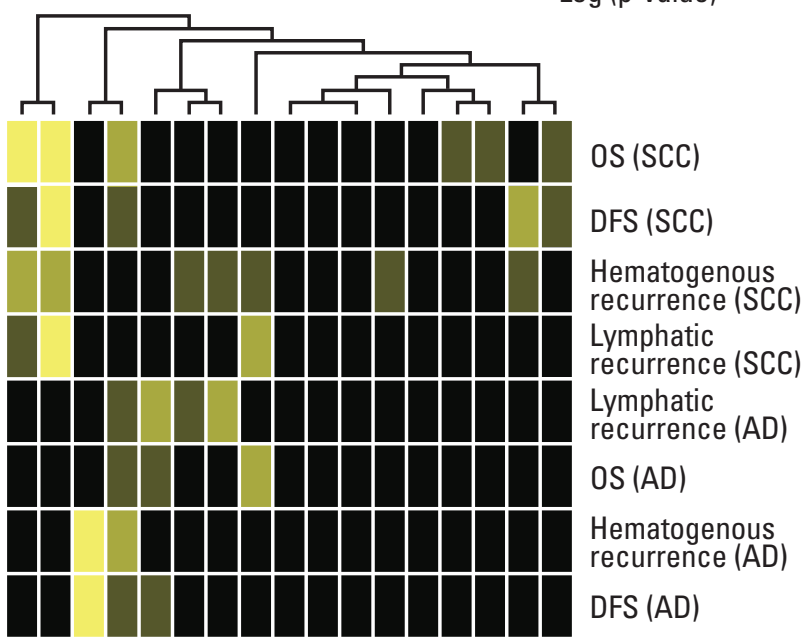

Color key and histogram

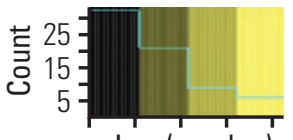

- Log (p-value)

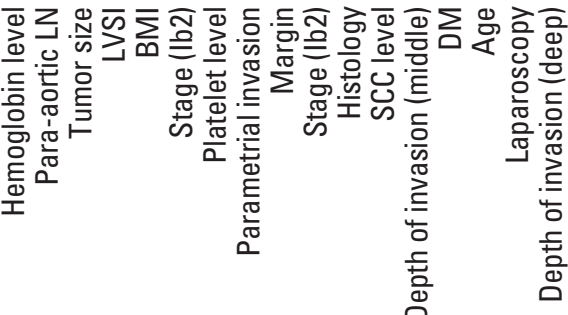

Fig. 4. (A) Histogram showing significance of risk factors for disease-free survival (DFS), hematogenous recurrence, lymphatic recurrence, and overall survival (OS). (B) Histogram showing significance of risk factors by histologic type. LN, lymph node; SCC, squamous cell carcinoma; DM, diabetes mellitus; LVSI, lymphovascular space invasion; BMI, body mass index; $\mathrm{AD}$, adenocarcinoma. 


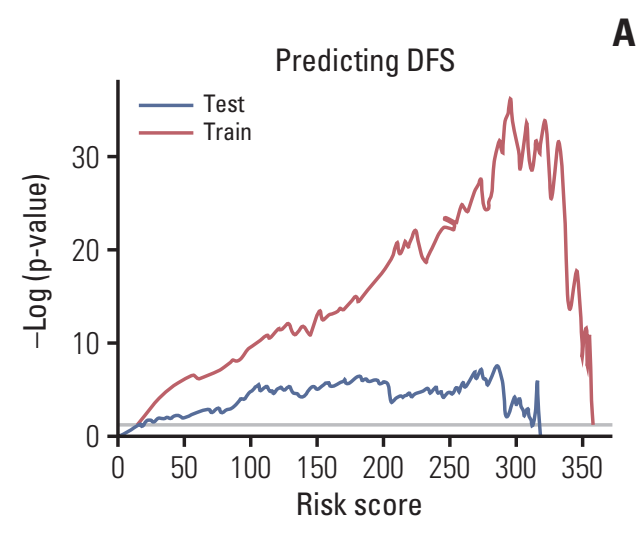

A
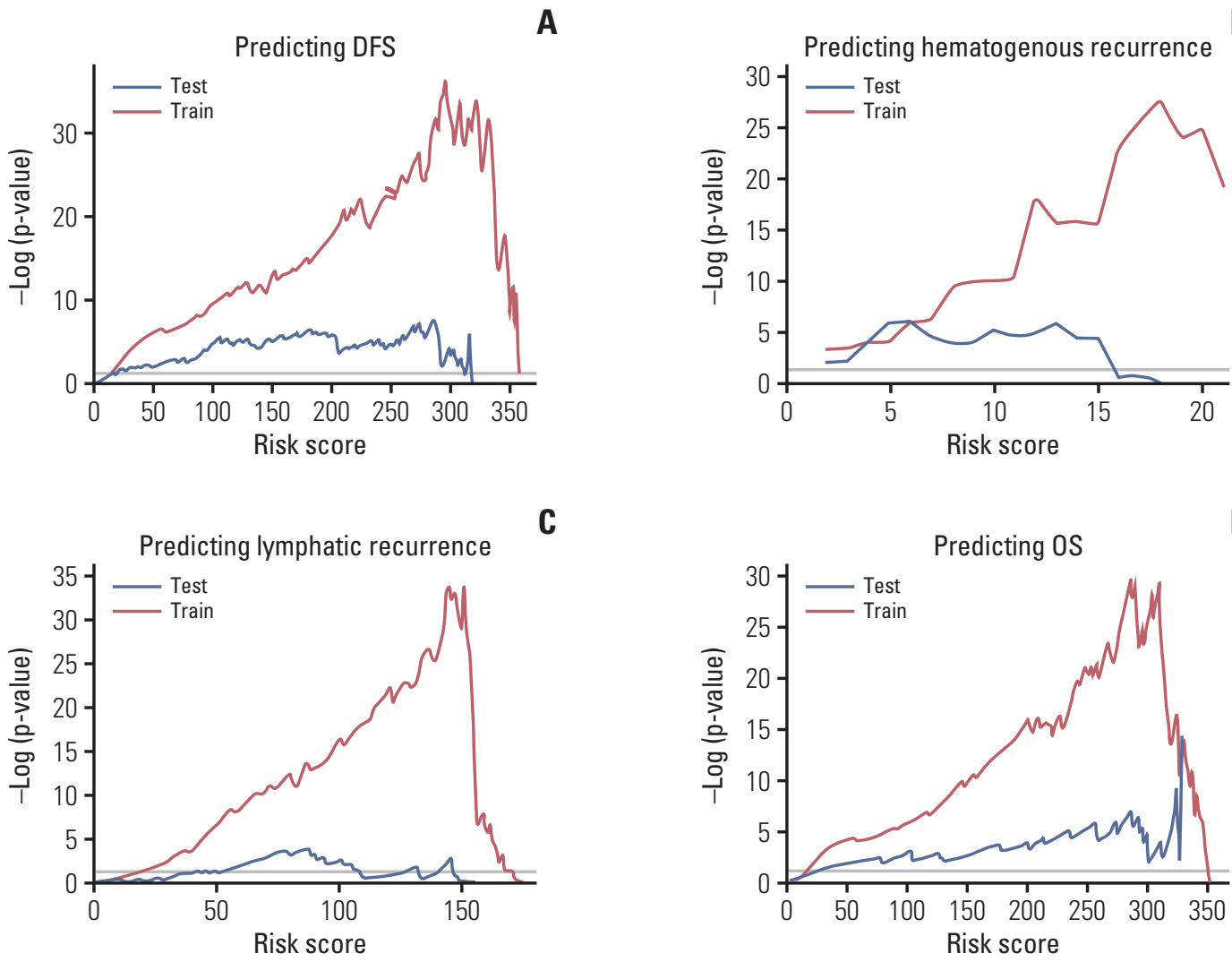

C

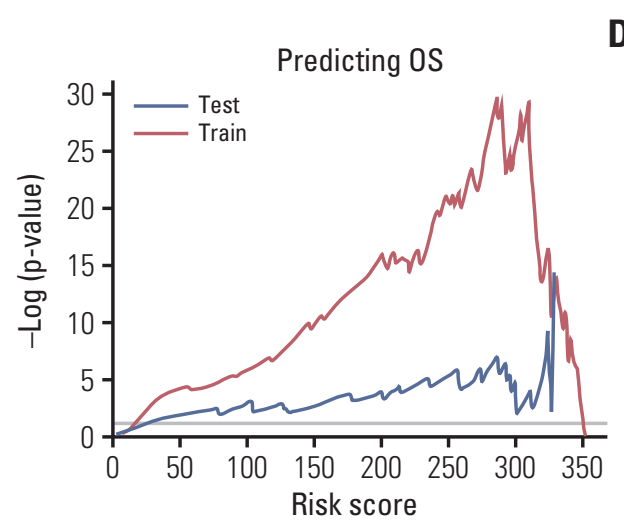

Fig. 5. Validation of the model predicting disease-free survival (DFS) (A), hematogenous recurrence (B), lymphatic recurrence (C), and overall survival (OS) (D).

of para-aortic lymph node metastasis (pa-LNM), histology, LVSI, DI, pelvic lymph node metastasis ( $\mathrm{p}-\mathrm{LNM})$, and pretreatment serum $\mathrm{Hb}$ level for DFS (RS=1.46×adenocarcinoma $+1.12 \times$ LVSI $+1.08 \times$ deep third DI $+0.63 \times \mathrm{p}-\mathrm{LNM}+2.16 \times$ pa-LNM-0.17×Hb) (Fig. 3A). Pa-LNM, histology, DI, LVSI, stage, and pretreatment serum $\mathrm{Hb}$ level were significant factors for OS (RS=0.78 $\times$ stage $+1.58 \times$ adenocarcinoma $+1.14 \times$ LVSI+1.21 $\times$ deep third DI+2.31 $\times$ pa-LNM-0.19 $\times \mathrm{Hb}$ ) (Fig. 3B). Pa-LNM, p-LNM, DI, and age were significant predictors for lymphatic recurrence $(\mathrm{RS}=1.89 \times$ deep third $\mathrm{DI}+2.27 \times \mathrm{p}-\mathrm{LNM}+$ 4.45×pa-LNM-0.71×age) (Fig. 3C). Histology, DI, pa-LNM, LVSI, and PMI were significant predictors of hematogenous recurrence $(\mathrm{RS}=1.92 \times$ adenocarcinoma $+1.11 \times \mathrm{LVSI}+1.69 \times$ deep third DI+1.61×pa-LNM+0.77×PMI) (Fig. 3D). Notably, stage was not significantly related to hematogenous recurrence, and histologic type was not correlated to lymphatic recurrence.

Additionally, for evaluation of prognostic factors in different histologic type, we performed separate analysis for SCC and adenocarcinoma patients of entire cohort. The relative prognostic significance of each factor was plotted in a heatmap, in which bright yellow indicates more significance (less p-value) (Fig. 4). Fig. 4A shows significance of factors in entire cohort, and Fig. 4B shows factors by different histologic type (SCC and adenocarcinoma). In SCC, but not in adenocarcinoma, pa-LNM was a significant risk factor for both DFS and OS (Fig. 4B). For DFS and hematogenous recurrence in adenocarcinoma, tumor size was a significant risk factor, different from SCC. Laparoscopy was shown in dark yellow (borderline significance) for DFS in SCC, but not in adenocarcinoma, in our data.

\section{Model validation}

For internal and external validation, we plotted p-values of the log-rank test using all possible cutoffs of RSs from each model. The p-values demonstrate the accurate performance of the model (Fig. 5). The validation plot (blue line) also showed good performance except for lymphatic recurrence. The prognostic model for lymphatic recurrence was not well validated in the external cohort.

The model is further validated by calculated probabilities of an event at 2 and 5 years for OS, DFS, hematogenous recurrence, and lymphatic recurrence using time-dependent 
A
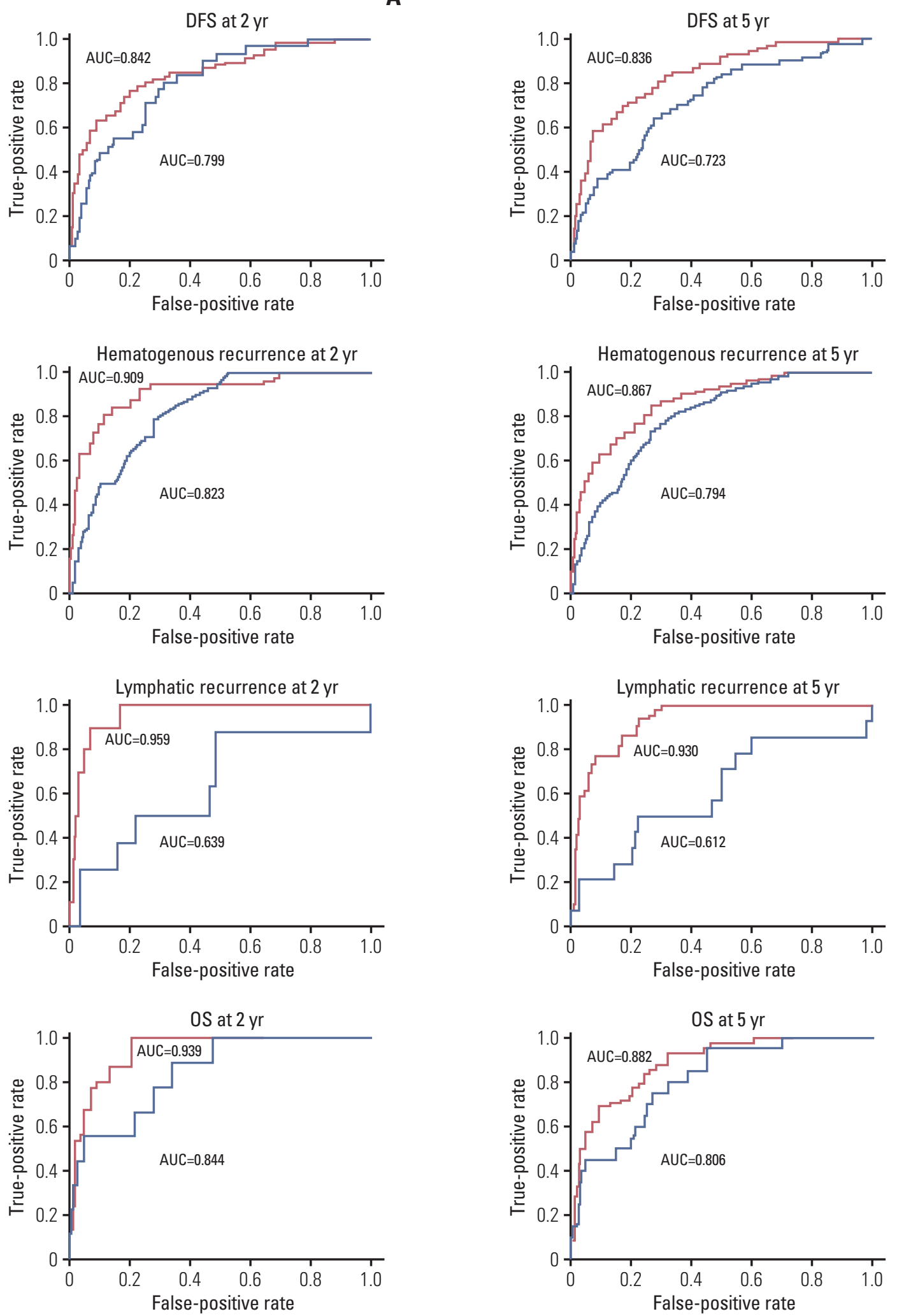

Fig. 6. Time-dependent receiver operating characteristic curves of the prediction model for disease-free survival (DFS), hematogenous recurrence, lymphatic recurrence, and overall survival (OS) (A, at 2 years; B, at 5 years). Red line indicates train cohort, and blue line indicates test cohort. AUC, area under the curve. 
Table 2. Characteristics of proposed model-classified risk groups for OS and DFS within patients group by traditional risk assessment in cervical cancer

\begin{tabular}{|c|c|c|c|c|c|c|}
\hline & \multicolumn{3}{|c|}{ Low risk by traditional risk } & \multicolumn{3}{|c|}{ High risk by traditional risk } \\
\hline & $\begin{array}{c}\text { Low risk by } \\
\text { proposed model }\end{array}$ & $\begin{array}{l}\text { High risk by } \\
\text { proposed model }\end{array}$ & p-value & $\begin{array}{c}\text { Low risk by } \\
\text { proposed model }\end{array}$ & $\begin{array}{l}\text { High risk by } \\
\text { proposed model }\end{array}$ & p-value \\
\hline OS & 542 & 9 & & 60 & 102 & \\
\hline \multicolumn{7}{|l|}{ Stage } \\
\hline Ib1/IIa & $535(98.7)$ & $7(77.8)$ & $<0.001$ & $59(98.3)$ & $50(49.0)$ & $<0.001$ \\
\hline $\mathrm{Ib} 2$ & $7(1.3)$ & $2(22.2)$ & & $5(8.3)$ & $52(51.0)$ & \\
\hline \multicolumn{7}{|l|}{ Histology } \\
\hline SCC & $511(94.3)$ & $1(11.1)$ & $<0.001$ & $59(98.3)$ & $59(57.8)$ & $<0.001$ \\
\hline $\mathrm{AD}$ & $31(5.7)$ & $8(88.9)$ & & $1(1.7)$ & $43(42.2)$ & \\
\hline \multicolumn{7}{|l|}{ LVSI } \\
\hline Negative & $469(86.5)$ & $5(55.6)$ & 0.030 & $32(53.3)$ & $4(3.9)$ & $<0.001$ \\
\hline Positive & 73 (13.5) & $4(44.4)$ & & $28(46.7)$ & $98(96.1)$ & \\
\hline \multicolumn{7}{|l|}{ Invasion depth } \\
\hline Inner $1 / 3$ & $309(57.0)$ & $3(33.3)$ & $<0.001$ & $17(28.3)$ & 0 & $<0.001$ \\
\hline Middle $1 / 3$ & $166(30.6)$ & 0 & & $26(43.3)$ & $3(2.9)$ & \\
\hline Deep $1 / 3$ & $67(12.4)$ & $6(66.7)$ & & $17(28.3)$ & $99(97.1)$ & \\
\hline \multicolumn{7}{|l|}{ Lymph node metastasis } \\
\hline Negative & $542(100)$ & $7(77.8)$ & NS & $17(28.3)$ & $16(15.7)$ & 0.009 \\
\hline Pelvic LN positive & 0 & 0 & & $43(71.7)$ & $75(73.5)$ & \\
\hline Para-aortic LN positive & 0 & $2(22.2)$ & & 0 & $11(10.8)$ & \\
\hline \multicolumn{7}{|l|}{ Parametrial involvement } \\
\hline Negative & $542(100)$ & $9(100)$ & NS & $46(76.7)$ & $51(50.0)$ & 0.001 \\
\hline Positive & 0 & 0 & & $14(23.3)$ & $51(50.0)$ & \\
\hline \multicolumn{7}{|l|}{ Resection margin } \\
\hline Negative & $539(99.4)$ & $9(100)$ & NS & $52(86.7)$ & $87(85.3)$ & 0.741 \\
\hline CIN positive & $3(0.6)$ & 0 & & 0 & $1(1.0)$ & \\
\hline Cancer positive & 0 & 0 & & $8(13.3)$ & $14(13.7)$ & \\
\hline DFS & 566 & 48 & & & & \\
\hline \multicolumn{7}{|l|}{ Stage } \\
\hline Ib1/IIa & $556(98.2)$ & $46(95.8)$ & 0.542 & $26(78.8)$ & $151(74.0)$ & 0.712 \\
\hline $\mathrm{Ib} 2$ & $10(1.8)$ & $2(4.2)$ & & $7(21.2)$ & $53(26.0)$ & \\
\hline \multicolumn{7}{|l|}{ Histology } \\
\hline SCC & $517(91.3)$ & $2(4.2)$ & $<0.001$ & $33(100)$ & $147(72.1)$ & 0.001 \\
\hline $\mathrm{AD}$ & $49(8.7)$ & $46(95.8)$ & & 0 & 57 (27.9) & \\
\hline \multicolumn{7}{|l|}{ LVSI } \\
\hline Negative & $493(87.1)$ & $31(64.6)$ & $<0.001$ & $27(81.8)$ & $11(5.4)$ & $<0.001$ \\
\hline Positive & 73 (12.9) & $17(35.4)$ & & $6(18.2)$ & $193(94.6)$ & \\
\hline \multicolumn{7}{|l|}{ Invasion depth } \\
\hline Inner $1 / 3$ & $318(56.2)$ & $16(33.3)$ & $<0.001$ & $8(24.2)$ & $2(1.0)$ & $<0.001$ \\
\hline Middle 1/3 & $176(31.1)$ & $5(10.4)$ & & $12(36.4)$ & $11(5.4)$ & \\
\hline Deep $1 / 3$ & $72(12.7)$ & $27(56.2)$ & & $13(39.4)$ & $191(93.6)$ & \\
\hline \multicolumn{7}{|l|}{ Lymph node metastasis } \\
\hline Negative & $566(100)$ & $45(93.8)$ & NS & $23(69.7)$ & $23(11.3)$ & $<0.001$ \\
\hline Pelvic LN positive & 0 & 0 & & $10(30.3)$ & $170(83.3)$ & \\
\hline Para-aortic LN positive & 0 & $3(6.2)$ & & 0 & $11(5.4)$ & \\
\hline \multicolumn{7}{|l|}{ Parametrial involvement } \\
\hline Negative & $566(100)$ & $48(100)$ & NS & 19 (57.6) & $126(61.8)$ & 0.791 \\
\hline Positive & 0 & 0 & & $14(42.4)$ & $78(38.2)$ & \\
\hline
\end{tabular}

(Continued to the next page) 
Table 2. Continued

\begin{tabular}{|c|c|c|c|c|c|c|}
\hline & \multicolumn{3}{|c|}{ Low risk by traditional risk } & \multicolumn{3}{|c|}{ High risk by traditional risk } \\
\hline & $\begin{array}{c}\text { Low risk by } \\
\text { proposed model }\end{array}$ & $\begin{array}{c}\text { High risk by } \\
\text { proposed model }\end{array}$ & p-value & $\begin{array}{c}\text { Low risk by } \\
\text { proposed model }\end{array}$ & $\begin{array}{c}\text { High risk by } \\
\text { proposed model }\end{array}$ & p-value \\
\hline \multicolumn{7}{|l|}{ Resection margin } \\
\hline Negative & $563(99.5)$ & $48(100)$ & NS & $23(69.7)$ & $180(88.2)$ & 0.018 \\
\hline CIN positive & $3(0.5)$ & 0 & & $1(3.0)$ & $2(1.0)$ & \\
\hline Cancer positive & 0 & 0 & & $9(27.3)$ & $22(10.8)$ & \\
\hline
\end{tabular}

Values are presented as number (\%). OS, overall survival; DFS, disease-free survival; SCC, squamous cell carcinoma; AD, adenocarcinoma; LVSI, lymphovascular space invasion; NS, not significant; LN, lymph node; CIN, cervical intraepithelial neoplasia.

ROC curves (Fig. 6). AUCs of train cohort (red line) and test cohort (blue line) are shown. In train cohort, AUCs of OS at 2 and 5 years were 0.939 and 0.882 . As in Fig. 6, except for the model for lymphatic recurrence, the proposed models were well validated in this ROC curve (AUCs in test cohort at 2 and 5 years were $0.799 / 0.723$ for DFS, $0.823 / 0.794$ for hematogenous recurrences, and 0.844/0.806 for OS, respectively).

\section{Comparison of prediction by traditional risks and the proposed model}

Our results indicate significant differences for histology, LVSI, and DI, none of which were traditionally seen as highrisk factors, between low and high-risk groups of proposed model within each traditional risk assessment group (Table 2). There is, therefore, a discrepancy between traditional risk factors and significant factors of the proposed model.

To compare prediction by traditional risks and the proposed model, RSs of DFS and OS by proposed model are plotted (blue dots for low risk, red dots for moderate risk, green dots for high risk of proposed model) according to traditional risk factor groups (e.g., low-risk factor, intermediate-risk factor, and high-risk factor) (Fig. 7A). Although there were correlations between groups by traditional risk factors and proposed model-classified risk groups, a number of patients with discordancy were observed. The Kaplan-Meier plot indicates that the proposed model-classified risk groups (Fig. 7C) showed more distinctive survival differences between each risk group than traditional risk groups (Fig. 7B).

\section{Discussion}

Several previous studies have attempted to identify prognostic factors in cervical cancer. However, these previous studies were limited by small patient numbers and retrospectively collected data [11,13,21-24]. Previous studies that evaluated the 12 prognostic models for early-stage cervical carcinoma underestimated DFS in all 12 models, and only a few of those models were applicable to other populations [25]. In a validation study of two prognostic models in 221 patients, Van de Putte et al. [26] concluded that both the prognostic index by Delgado et al. [3] and the classification by Sedlis et al. [5] could be applied to their patient groups. Compared to previous studies, our study is robust because of our use of a comparatively large number of patients treated in multiple institutions. The treatment guideline was nearly unchanged during the study time period, which was beneficial to this work. Additionally, we performed external validation using a separate data set, which is essential for general applicability of a predictive model.

There have been efforts to improve patients' survival outcomes with the use of adjuvant radiation (with or without chemotherapy). However, only the significant change in decreasing recurrence was shown, but not in survival $[5,27]$. Prognostic indicators from pathologic findings in cervical cancer include histologic cell type, tumor size, tumor grade, LVSI, depth of cervical stromal invasion, surgical margins, and lymph node metastases status [27,28]. Identifying risk factors for patients with early-stage cervical cancer is an important step for determination of adequate adjuvant therapy after radical hysterectomy. The prognosis of patients is affected by a number of risk factors that interact with each other. This may be related to the diversity of predictive value from various prognostic variables.

In contrast to traditional prognostic systems, which allocate patients into discrete risk groups, a statistical predictive 

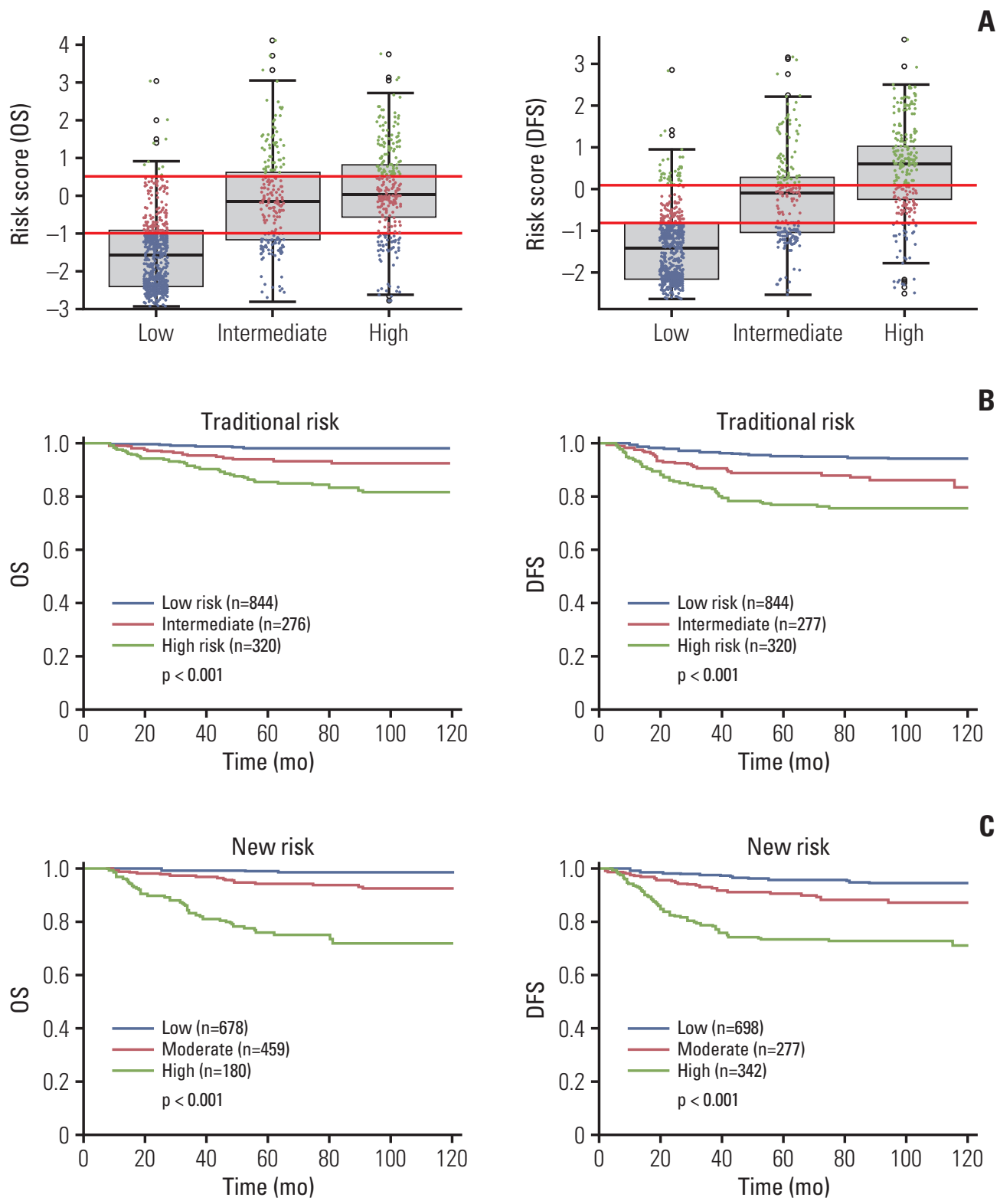

Fig. 7. Comparison of traditional risk groups and proposed model-classified risk groups and their prognostic significance. (A) Box plot of risk score according to traditional risk assessment. (B) Kaplan-Meier survival according to traditional risk groups. (C) Kaplan-Meier curve according to proposed model-classified risk groups. OS, overall survival; DFS, disease-free survival.

model can generate a numerical probability of a clinical outcome. Combining weighted clinicopathologic variables, the proposed model can give more individualized predictions. It is not surprising that a predictive model performs better than the traditional staging system because it is better able to account for heterogeneity in tumor and patient characteristics. Another potential benefit of the predictive statistical model is that it is readily modifiable by adding other prognostic factors. Here, we report a discrepancy between tradi- tional risk factors and proposed model-classified risks. Factors not expected to be high risk based on traditional risk assessment-such as histology, LVSI, and DI-showed significant differences for OS, DFS, and hematogenous recurrence in groups traditionally characterized as both low and high risk. Also, in traditional high-risk group, LNM, size, and pretreatment serum $\mathrm{Hb}$ level showed significant differences for OS and DFS between groups by proposed model-classified risks. 
A recent phase 3, multicenter, prospective randomized trial, the Laparoscopic Approach to Cervical Cancer (LACC) Trial, concluded that laparoscopic radical hysterectomy in patients with cervical cancer was associated with lower rates of DFS and OS than open radical hysterectomy [29]. Similarly in our data, laparoscopy was associated with DFS with borderline significance in SCC. However, laparoscopy was not significant factor in adenocarcinoma. In LACC trial, histologic subtype of SCC was most common (with 67.1\%-67.3\%) compared to adenocarcinoma (25.6\%-27.3\%), and result would be mostly affected by squamous cell carcinoma. We could expect different outcomes between histologic types, and further study of adenocarcinoma is needed.

Although we constructed an improved prognostic model, there are a number of potential limitations in the current study that must be acknowledged. First, we included patients who received adjuvant therapy in the study population. The vast majority of literature supported that adjuvant radiotherapy or concurrent chemoradiotherapy may reduce the risk of recurrence in patients with cervical cancer with adverse risk factors $[5,6]$. Therefore, the adjuvant therapy might blunt the effect of prognostic variables. The patient cohorts in this study may not represent the whole population with early-stage cervical cancer. For example, our sampled cohort had 5-year OS of $95.5 \%$, which is considerably different than reports in other studies, such as an OS of 62.8\% [30]. Adjuvant treatments may, in part, explain the remarkably good survival rates in this study. To overcome the limit of the study population, validation of the model in a less favorable prognostic group is necessary before it can be adopted more broadly. Our model was created with retrospectively collected data. Therefore, our study may have biases with patient selection, deficit of random assignment, and incomplete data collection. Prospective data collection could identify more definite prognostic factors that could explain outcomes more precisely. The use of multi-institutional study data could be considered as a limitation because multiple surgeons and pathologists from different institutions may not rely on the same treatment and management for cervical cancer patients. Other differences from the multiple centers may also exist. However, the use of multi-institutional study data could also be regarded as an advantage of our study, as our conclusions could be more generally applicable. Also, large sample sizes are important for studies focused on a patient group with a low rate of the event being studied. Recently, FIGO staging system for cervical cancer was revised. Notable difference would be increased sub-stage in IB disease by tumor size, and incorporation of nodal status in staging. We inevitably used prior 2014 FIGO staging, because analysis was had been performed with retrospectively collected data. At this point, confusion may occur due to using prior FIGO staging, and this could also be limitation of our study.

In conclusion, we developed a robust model that can predict the OS and DFS in patients with early-stage cervical cancer after surgery. The model was internally and externally validated. This model may provide more individualized predictions in clinical practice for early-stage cervical cancer. A prospective validation study in a population with diverse characteristics may be needed to improve the predictive accuracy of the model in the future.

\section{Conflicts of Interest}

Conflict of interest relevant to this article was not reported.

\section{Acknowledgments}

We thank all patients and their families, the investigators and study teams at the participating sites.

\section{Author Details}

${ }^{1}$ Department of Obstetrics and Gynecology, Samsung Medical Center, Sungkyunkwan University School of Medicine, Seoul, ${ }^{2}$ Cancer Healthcare Research Branch, Center for Uterine Cancer, and Center for Clinical Trials, Research Institute and Hospital and Cancer Control and Policy, Graduate School of Cancer Science and Policy, National Cancer Center, Goyang, '3epartment of Obstetrics and Gynecology, Korea Cancer Center Hospital, Korea Institute of Radiological and Medical Sciences, Seoul, ${ }^{4}$ Department of Obstetrics and Gynecology, Ewha Womans University Mokdong Hospital, Ewha Womans University School of Medicine, Seoul, ${ }^{5}$ Medical Treatment Division, Gwangjin-gu Health Center, Seoul, 'Department of Obstetrics and Gynecology, CHA Gangnam Medical Center, CHA University, Seoul, 'Department of Obstetrics and Gynecology, Seoul National University Bundang Hospital, Seongnam, ${ }^{8}$ Department of Obstetrics and Gynecology, Kyung Hee University Hospital at Gangdong, Kyung Hee University School of Medicine, Seoul, ${ }^{9}$ Department of Obstetrics and Gynecology, Sanggye Paik Hospital, Inje University College of Medicine, Seoul, Korea 


\section{References}

1. Ferlay J, Soerjomataram I, Dikshit R, Eser S, Mathers C, Rebelo $\mathrm{M}$, et al. Cancer incidence and mortality worldwide: sources, methods and major patterns in GLOBOCAN 2012. Int J Cancer. 2015;136:E359-86.

2. Lee WC, Lee SY, Koo YJ, Kim TJ, Hur SY, Hong SR, et al. Establishment of a Korea HPV cohort study. J Gynecol Oncol. 2013;24:59-65.

3. Delgado G, Bundy B, Zaino R, Sevin BU, Creasman WT, Major F. Prospective surgical-pathological study of disease-free interval in patients with stage IB squamous cell carcinoma of the cervix: a Gynecologic Oncology Group study. Gynecol Oncol. 1990;38:352-7.

4. Landoni F, Maneo A, Colombo A, Placa F, Milani R, Perego P, et al. Randomised study of radical surgery versus radiotherapy for stage Ib-IIa cervical cancer. Lancet. 1997;350:535-40.

5. Sedlis A, Bundy BN, Rotman MZ, Lentz SS, Muderspach LI, Zaino RJ. A randomized trial of pelvic radiation therapy versus no further therapy in selected patients with stage IB carcinoma of the cervix after radical hysterectomy and pelvic lymphadenectomy: a Gynecologic Oncology Group study. Gynecol Oncol. 1999;73:177-83.

6. Peters WA 3rd, Liu PY, Barrett RJ 2nd, Stock RJ, Monk BJ, Berek JS, et al. Concurrent chemotherapy and pelvic radiation therapy compared with pelvic radiation therapy alone as adjuvant therapy after radical surgery in high-risk early-stage cancer of the cervix. J Clin Oncol. 2000;18:1606-13.

7. Barter JF, Soong SJ, Shingleton HM, Hatch KD, Orr JW Jr. Complications of combined radical hysterectomy-postoperative radiation therapy in women with early stage cervical cancer. Gynecol Oncol. 1989;32:292-6.

8. Lagasse LD, Creasman WT, Shingleton HM, Ford JH, Blessing JA. Results and complications of operative staging in cervical cancer: experience of the Gynecologic Oncology Group. Gynecol Oncol. 1980;9:90-8.

9. Sevin BU, Nadji M, Lampe B, Lu Y, Hilsenbeck S, Koechli OR, et al. Prognostic factors of early stage cervical cancer treated by radical hysterectomy. Cancer. 1995;76(10 Suppl):1978-86.

10. Soisson AP, Soper JT, Clarke-Pearson DL, Berchuck A, Montana G, Creasman WT. Adjuvant radiotherapy following radical hysterectomy for patients with stage IB and IIA cervical cancer. Gynecol Oncol. 1990;37:390-5.

11. Kristensen GB, Abeler VM, Risberg B, Trop C, Bryne M. Tumor size, depth of invasion, and grading of the invasive tumor front are the main prognostic factors in early squamous cell cervical carcinoma. Gynecol Oncol. 1999;74:245-51.

12. Zaino RJ, Ward S, Delgado G, Bundy B, Gore H, Fetter G, et al. Histopathologic predictors of the behavior of surgically treated stage IB squamous cell carcinoma of the cervix. A Gynecologic Oncology Group study. Cancer. 1992;69:1750-8.

13. Tsai CS, Lai CH, Wang CC, Chang JT, Chang TC, Tseng CJ, et al. The prognostic factors for patients with early cervical cancer treated by radical hysterectomy and postoperative radiotherapy. Gynecol Oncol. 1999;75:328-33.

14. Ryu SY, Kim MH, Nam BH, Lee TS, Song ES, Park CY, et al.
Intermediate-risk grouping of cervical cancer patients treated with radical hysterectomy: a Korean Gynecologic Oncology Group study. Br J Cancer. 2014;110:278-85.

15. Abu-Rustum NR, Zhou Q, Gomez JD, Alektiar KM, Hensley ML, Soslow RA, et al. A nomogram for predicting overall survival of women with endometrial cancer following primary therapy: toward improving individualized cancer care. Gynecol Oncol. 2010;116:399-403.

16. Ho CM, Chien TY, Huang SH, Wu CJ, Shih BY, Chang SC. Multivariate analysis of the prognostic factors and outcomes in early cervical cancer patients undergoing radical hysterectomy. Gynecol Oncol. 2004;93:458-64.

17. Lai CH, Chang CJ, Huang HJ, Hsueh S, Chao A, Yang JE, et al. Role of human papillomavirus genotype in prognosis of early-stage cervical cancer undergoing primary surgery. J Clin Oncol. 2007;25:3628-34.

18. Yuan CC, Wang PH, Lai CR, Yen MS, Chen CY, Juang CM. Prognosis-predicting system based on factors related to survival of cervical carcinoma. Int J Gynaecol Obstet. 1998;63: 163-7.

19. Greenland S, Schwartzbaum JA, Finkle WD. Problems due to small samples and sparse data in conditional logistic regression analysis. Am J Epidemiol. 2000;151:531-9.

20. Heagerty PJ, Lumley T, Pepe MS. Time-dependent ROC curves for censored survival data and a diagnostic marker. Biometrics. 2000;56:337-44.

21. Sevin BU, Lu Y, Bloch DA, Nadji M, Koechli OR, Averette HE. Surgically defined prognostic parameters in patients with early cervical carcinoma: a multivariate survival tree analysis. Cancer. 1996;78:1438-46.

22. Smiley LM, Burke TW, Silva EG, Morris M, Gershenson DM, Wharton JT. Prognostic factors in stage IB squamous cervical cancer patients with low risk for recurrence. Obstet Gynecol 1991;77:271-5.

23. Ishikawa $H$, Nakanishi T, Inoue T, Kuzuya K. Prognostic factors of adenocarcinoma of the uterine cervix. Gynecol Oncol. 1999;73:42-6.

24. Odell EW, Jani P, Sherriff M, Ahluwalia SM, Hibbert J, Levison DA, et al. The prognostic value of individual histologic grading parameters in small lingual squamous cell carcinomas. The importance of the pattern of invasion. Cancer. 1994;74:789-94.

25. Biewenga P, van der Velden J, Mol BW, Stalpers LJ, Schilthuis MS, van der Steeg JW, et al. Validation of existing prognostic models in patients with early-stage cervical cancer. Gynecol Oncol. 2009;115:277-84.

26. Van de Putte G, Lie AK, Vach W, Baekelandt M, Kristensen GB. Risk grouping in stage IB squamous cell cervical carcinoma. Gynecol Oncol. 2005;99:106-12.

27. Aoki Y, Sasaki M, Watanabe M, Sato T, Tsuneki I, Aida H, et al. High-risk group in node-positive patients with stage IB, IIA, and IIB cervical carcinoma after radical hysterectomy and postoperative pelvic irradiation. Gynecol Oncol. 2000;77: 305-9.

28. Lai CH, Hong JH, Hsueh S, Ng KK, Chang TC, Tseng CJ, et al. 
Preoperative prognostic variables and the impact of postoperative adjuvant therapy on the outcomes of Stage IB or II cervical carcinoma patients with or without pelvic lymph node metastases: an analysis of 891 cases. Cancer. 1999;85:1537-46.

29. Ramirez PT, Frumovitz M, Pareja R, Lopez A, Vieira M, Ribeiro R, et al. Minimally invasive versus abdominal radical hysterectomy for cervical cancer. N Engl J Med. 2018;379:1895904.

30. Benard VB, Watson M, Saraiya M, Harewood R, Townsend JS, Stroup AM, et al. Cervical cancer survival in the United States by race and stage (2001-2009): findings from the CONCORD2 study. Cancer. 2017;123:5119-37. 